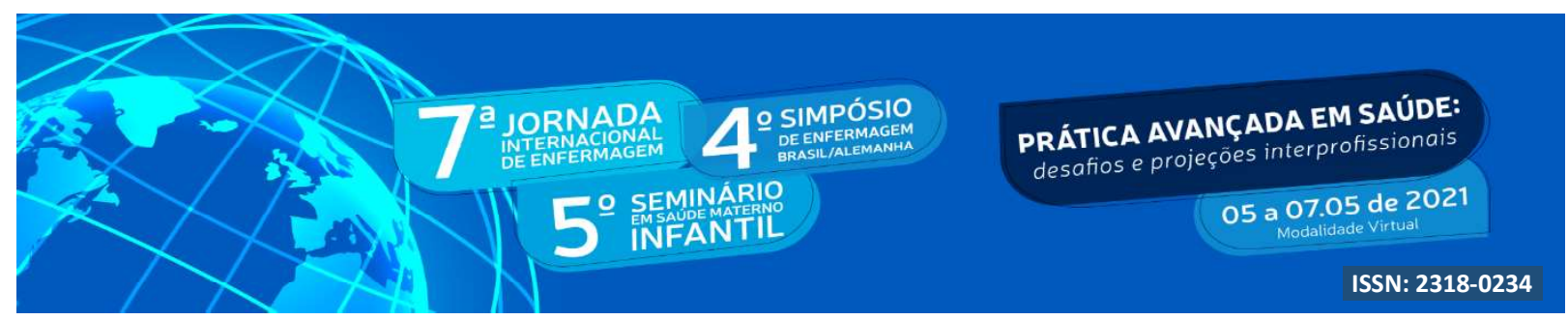

DOI: http://doi.org/10.48195/jie2021-180

\title{
SISTEMATIZAÇÃO DA ASSISTÊNCIA DE ENFERMAGEM: PARA ALÉM DOS PRECEITOS LEGAIS ${ }^{1}$
}

\author{
Aline Medianeira Gomes Correa ${ }^{2}$;isiane de Borba Müller ${ }^{3}$ \\ Elviani Santos Stefano ${ }^{4}$;SilomarIlha ${ }^{5}$;Dirce Stein Backes ${ }^{6}$.
}

\section{RESUMO}

Objetivo: Conhecer a percepção de Enfermeiros atuantes em Unidade Intensiva Pediátrica e Neonatal sobre a Sistematização da Assistência de Enfermagem. Método: Pesquisa exploratório-descritiva, qualitativa, realizada com 15 enfermeiros que atuam em Unidade Intensiva Pediátrica e Neonatal de um hospital de médio porte da região leste do Estado do Rio Grande do Sul, a partir de entrevistas com questões norteadoras. Os dados coletados entre março e julho de 2017, com uma entrevista semiestruturada, foram submetidos à análise de conteúdo. Resultados: Evidenciaram-se duas categorias: Sistematização da Assistência de Enfermagem: processo legal e privativo do Enfermeiro; Sistematização da Assistência de Enfermagem: da concepção à prática assistencial. Conclusão: Os Enfermeiros das unidades intensivas pediátrica/neonatal possuem conhecimento teórico superficial sobre à proposta teórico-metodológica da Sistematização da Assistência de Enfermagem. Para a maioria, a Sistematização limita-se no cumprimento dos preceitos legais e na execução das etapas do diagnóstico e das intervenções de enfermagem.

Palavras-chave:Cuidados de enfermagem; Neonatologia; Processo de enfermagem; Pediatria; Unidades de Terapia Intensiva.

\begin{abstract}
Objective: To know the perception of Nurses working in Pediatric and Neonatal Intensive Unit on the Systematization of Nursing Care. Method: A qualitative, exploratory-descriptive study carried out with 15 nurses who work in the Pediatric and Neonatal Intensive Unit of a mid-size hospital in the eastern region of the State of Rio Grande do Sul, based on interviews with guiding questions. The data collected between March and July 2017, with a semi-structured interview, were submitted to content analysis. Results: Two categories were evidenced: Systematization of Nursing Assistance: legal and private process of Nursing; Systematization of nursing care: from conception to care practice. Conclusion: The nurses of the pediatric / neonatal intensive units have superficial theoretical

\footnotetext{
${ }^{2}$ Enfermeira.Egressa do Mestrado Profissional em Saúde Materno Infantil da UFN E mail:alinegc@yahoo.com.br.

${ }^{3}$ Enfermeira.Egressa do Mestrado Profissional em Saúde Materno Infantil da UFN. Email:lisi_enf@yahoo.com.br.

${ }^{4}$ Enfermeira.Mestranda em Ciências da saúde e da vida da Universidade Franciscana.UFN. Email:stfelvi@yahoo.com.br.

${ }^{5}$ Enfermeiro.Docente do Curso de Enfermagem e do Mestrado Acadêmico em Ciências da Saúde e da Vida da UFN. E-mail:silomar.ilha@ufn.edu.br.

${ }^{6}$ Orientadora.Doutora em Enfermagem. Docente do Curso de Enfermagem e Coordenadora do Mestrado Profissional Saúde Materno Infantil - Universidade Franciscana. E-mail:backesdirce@ufn.edu.br
} 


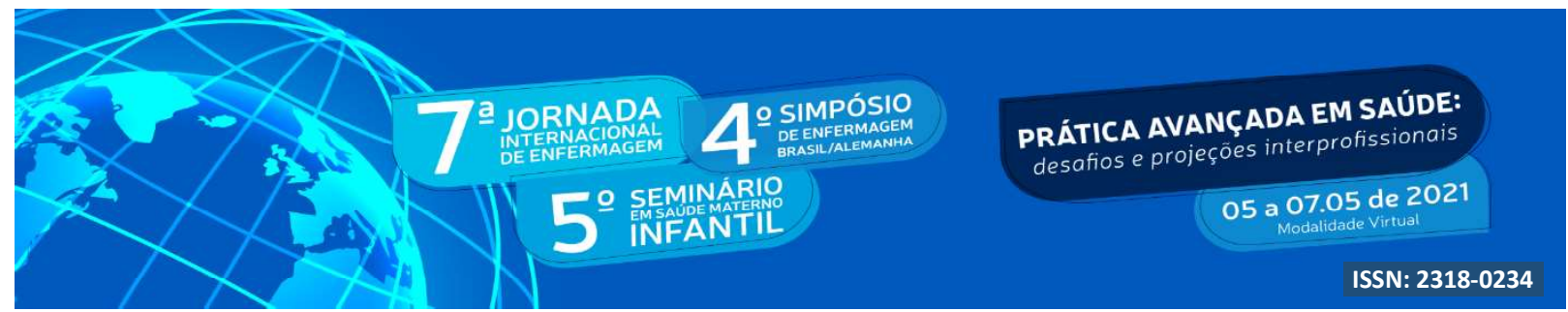

knowledge about the theoretical-methodological proposal of Nursing Care Systematization. For the majority, Systematization is limited in compliance with legal precepts and in the execution of the stages of diagnosis and nursing interventions.

Key Words: Nursing care; Neonatology; Nursing Process; Pediatrics; Intensive care units.

\section{INTRODUÇÃO}

A Sistematização da Assistência de Enfermagem (SAE) foi regulamentada, no Brasil, como método científico para organizar e otimizar a gestão e a assistência por meio da operacionalização do Processo de Enfermagem (PE). A SAE tem como propósito assegurar uma assistência singular, multidimensional e de qualidade, a partir de cinco etapas interrelacionadas e complementares, quais sejam: coleta de dados, diagnóstico de enfermagem, planejamento, implementação e avaliação de enfermagem (COFEN, 2009).

$\mathrm{Na}$ busca pela garantia da qualidade da assistência de enfermagem e de uma maior visibilidade do papel gerencial e assistencial do Enfermeiro, o Conselho Federal de Enfermagem (COFEN) instituiu, no ano de 2002, por meio da Resolução COFEN no 272/2002, a obrigatoriedade da implementação da SAE em todos os serviços de saúde públicos e privados, nos quais atuam profissionais de enfermagem (COFEN, 2002). Esta Resolução foi revogada, na sequência, pela Resolução COFEN no 358/2009, que além de determinar a implementação da SAE na prática cotidiana dos serviços de enfermagem, a preconiza como atividade privativa e proativa do enfermeiro, baseada em estratégias metodológicas científicas que vão desde o planejamento à avaliação do processo de enfermagem (COFEN, 2009).

Estudos realizados, nesta área, demonstram que a SAE tem forte potencial para assegurar a qualidade da assistência de enfermagem, bem como ampliar a visibilidade profissional e fortalecer a clínica ampliada do Enfermeiro, pela (re)definição de seu espaço de atuação e de sua conduta com base em ferramentas científicas (OLIVEIRA,MEDEIROS, 2012).Nessa

${ }^{2}$ Enfermeira.Egressa do Mestrado Profissional em Saúde Materno Infantil da UFN E mail:alinegc@yahoo.com.br.

${ }^{3}$ Enfermeira.Egressa do Mestrado Profissional em Saúde Materno Infantil da UFN. Email:lisi_enf@yahoo.com.br.

${ }^{4}$ Enfermeira.Mestranda em Ciências da saúde e da vida da Universidade Franciscana.UFN. Email:stfelvi@yahoo.com.br.

${ }^{5}$ Enfermeiro.Docente do Curso de Enfermagem e do Mestrado Acadêmico em Ciências da Saúde e da Vida da UFN. E-mail:silomar.ilha@ufn.edu.br.

${ }^{6}$ Orientadora.Doutora em Enfermagem. Docente do Curso de Enfermagem e Coordenadora do Mestrado Profissional Saúde Materno Infantil - Universidade Franciscana. E-mail:backesdirce@ufn.edu.br 


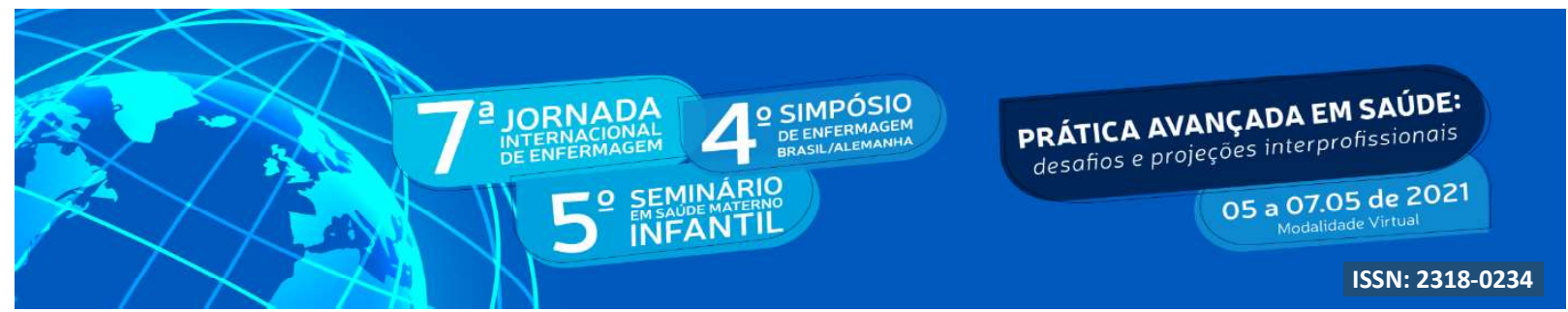

mesma direção, outros estudos evidenciaram que o enfermeiro enfrenta, ainda, importantes fragilidades e desafios na implementação da SAE, os quais não se reduzem a atuação técnica pontual, mas à compreensão ampliada e sistêmica da mesma como processo teórico-prático que visa tanto à dimensão singular do indivíduo, quanto à dimensão organizacional e gerencial do cuidado (SILVA, SOUZA et al, 2015).

Os desafios mencionados anteriormente são, ainda, maiores quando relacionados a implementação da SAE em unidades intensivas pediátricas. Estudo que objetivou avaliar o processo de implantação da SAE em uma unidade pediátrica de um hospital universitário evidenciou que o seu processo de implantação enfrenta barreiras que vão desde a sobrecarga de trabalho dos enfermeiros, à pouca apropriação e pouco envolvimento dos profissionais da equipe no processo de efetivação da SAE. O mesmo estudo evidenciou, ainda, que a inadequação e o não preenchimento dos formulários institucionais relacionados à $\mathrm{SAE}$ e a falta de articulação entre as fases do processo de enfermagem também foram fatores que dificultaram a implantação da SAE nessa unidade(TAVARES ,et al 2013).

Frente ao exposto, torna-se necessário conhecer a percepção dos enfermeiros sobre a SAE e sua disposição em utilizá-la na prática, uma vez que este profissional é o protagonista na implementação desta metodologia de trabalho e a forma como pensam e refletem repercute diretamente no modo como conduzem o seu processo de trabalho, fato que justifica a necessidade e relevância desse estudo. Na tentativa de ampliar o processo de investigação sobre a temática em questão e de contribuir para a consolidação da SAE, na prática, questiona-se: qual a percepção de Enfermeiros atuantes em Unidades Intensivas Pediátrica e Neonatal sobre a Sistematização da Assistência de Enfermagem?

$\mathrm{Na}$ tentativa de responder o questionamento este estudo objetivou conhecer a percepção de Enfermeiros atuantes em Unidades Intensivas Pediátrica e Neonatal sobre a Sistematização da Assistência de Enfermagem.

\footnotetext{
${ }^{2}$ Enfermeira.Egressa do Mestrado Profissional em Saúde Materno Infantil da UFN E mail:alinegc@yahoo.com.br.

${ }^{3}$ Enfermeira.Egressa do Mestrado Profissional em Saúde Materno Infantil da UFN. Email:lisienf@yahoo.com.br.

${ }^{4}$ Enfermeira.Mestranda em Ciências da saúde e da vida da Universidade Franciscana.UFN. Email:stfelvi@yahoo.com.br.

${ }^{5}$ Enfermeiro.Docente do Curso de Enfermagem e do Mestrado Acadêmico em Ciências da Saúde e da Vida da UFN. E-mail:silomar.ilha@ufn.edu.br.

${ }^{6}$ Orientadora.Doutora em Enfermagem. Docente do Curso de Enfermagem e Coordenadora do Mestrado Profissional Saúde Materno Infantil - Universidade Franciscana. E-mail:backesdirce@ufn.edu.br
} 


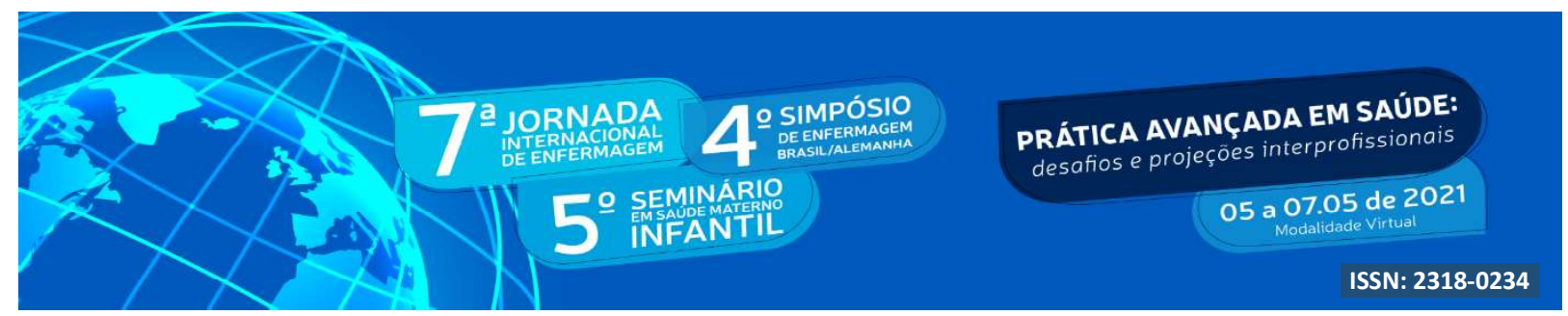

\section{OBJETIVO}

$\mathrm{Na}$ tentativa de responder o questionamento este estudo objetivou conhecer a percepção de Enfermeiros atuantes em Unidades Intensivas Pediátrica e Neonatal sobre a Sistematização da Assistência de Enfermagem.

\section{METODOLOGIA}

Trata-se de uma pesquisa exploratório-descritiva, de caráter qualitativo ${ }^{(9-10)}$, desenvolvida com enfermeiros que atuavam em Unidade Intensiva Pediátrica e neonatal de um hospital de médio porte da região leste do Estado do Rio Grande do Sul. O referido hospital é de caráter púbico, com 100\% de atendimento pelo Sistema Único de Saúde (SUS). Dispõe de uma Unidade de Terapia Intensiva (UTI) Neonatal e de uma Unidade de Cardiologia Intensiva (UCI) Pediátrica, ambas com 10 leitos de internação. A UTI neonatal conta com oito enfermeiros e a UCI pediátrica, com sete enfermeiros, totalizando 15 profissionais enfermeiros atuantes em unidades Intensivas Pediátrica e Neonatal.

Os critérios de inclusão para o estudo foram: ser enfermeiro e atuar ou já ter atuado na Unidade Intensiva Pediátrica ou Neonatal por um período mínimo de seis meses. Tempo suficiente para que os participantes já tenham vivenciado a rotina de trabalho nas referidas unidades, posto em prática a SAE, estando aptos a descrever suas vivências. Como critérios de exclusão, considerou-se: os enfermeiros que se encontravam em férias, atestado e/ou folgas nos dias e horários previamente agendados para a coleta de dados. Todos os profissionais atenderam aos critérios de inclusão, formando o corpus desse estudo os 15 enfermeiros atuantes nas referidas unidades.

O convite aos participantes ocorreu presencialmente e de forma individual, durante o turno de trabalho dos mesmos. Após o aceite em participar da pesquisa, foi agendado conforme a disponibilidade de cada participante o dia, local e a hora para a realização da coleta dos dados. Os dados foram coletados entre os meses de março e julho de 2017, por meio de entrevista semiestruturada com questões norteadoras, quais sejam: O que você ${ }^{2}$ Enfermeira.Egressa do Mestrado Profissional em Saúde Materno Infantil da UFN E mail:alinegc@yahoo.com.br.

${ }^{3}$ Enfermeira.Egressa do Mestrado Profissional em Saúde Materno Infantil da UFN. Email:lisi_enf@yahoo.com.br.

${ }^{4}$ Enfermeira.Mestranda em Ciências da saúde e da vida da Universidade Franciscana.UFN. Email:stfelvi@yahoo.com.br.

${ }^{5}$ Enfermeiro.Docente do Curso de Enfermagem e do Mestrado Acadêmico em Ciências da Saúde e da Vida da UFN. E-mail:silomar.ilha@ufn.edu.br.

${ }^{6}$ Orientadora.Doutora em Enfermagem. Docente do Curso de Enfermagem e Coordenadora do Mestrado Profissional Saúde Materno Infantil - Universidade Franciscana. E-mail:backesdirce@ufn.edu.br 


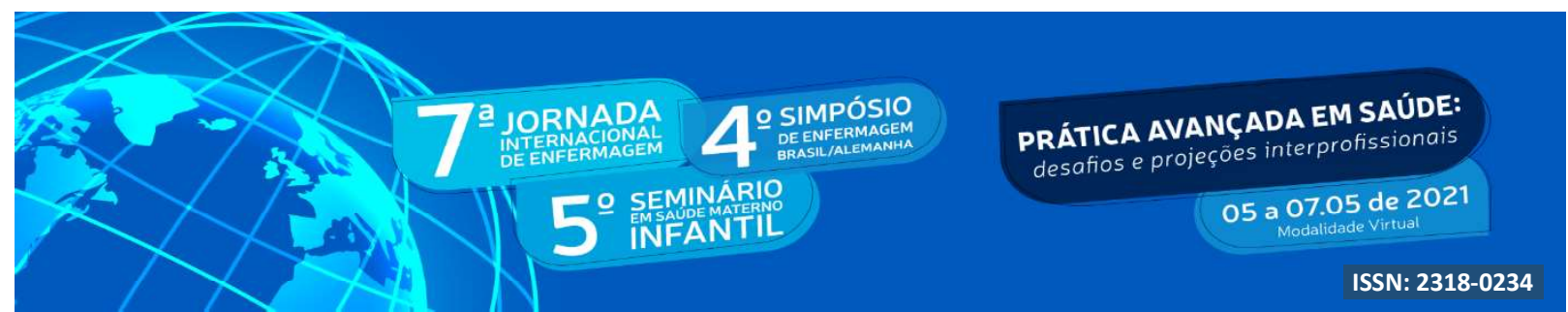

entende por Sistematização da Assistência de Enfermagem? Como você realiza a implementação da SAE na prática assistencial? As entrevistas foram gravadas em aparelho MP3 e, a seguir, transcritas pelo pesquisador principal para fins de análise dos dados.

O tratamento dos dados ocorreu com base na análise de conteúdo temática, a qual se constitui em três etapas. Na primeira etapa, denominada de pré-análise foi realizada uma leitura exaustiva dos dados, seguida da organização do material e formulação de hipóteses. A seguir foi feita a exploração do material, e a codificados os dados brutos. Na terceira e última etapa, os dados foram interpretados e delimitados em eixos temáticos pela compreensão dos significados estabelecidos (BARDIN, 2011).

O presente estudo foi submetido à apreciação do Comitê de Ética em Pesquisa com Seres Humanos (CEP), via Plataforma Brasil sistema CEP/CONEP. Somente após a aprovação do CEP foi realizado o primeiro contato com os participantes. Foram considerados os preceitos éticos que envolvem a pesquisa com seres humanos, conforme a Resolução 466/2012 do Ministério da Saúde (Ministério da Saúde; 2012).

O Projeto foi aprovado por Comitê de Ética em Pesquisa. Manteve-se o anonimato dos participantes, os quais foram identificandos, pela letra "E" (Enfermeiro), seguida de um algarismo numérico (E1, E2...E15).

\section{RESULTADOS E DISCUÇÃO}

Dos 15 profissionais participantes do estudo, 12 eram do sexo feminino e três, do masculino, com idades entre 27 e 47 anos. Destes, oito atuavam na UTI Neonatal e sete na UCI Pediátrica; nove possuíam especialização e seis eram generalistas.

Quanto ao tempo de formação, variou de quatro a 16 anos. Quanto ao tempo em que atuavam ou que já haviam atuado na unidade intensiva pediátrica e Neonatal, variou de um a nove anos. Os dados organizados e codificados pela análise de conteúdo resultaram em duas categorias temáticas, quais sejam: Sistematização da Assistência de Enfermagem:

\footnotetext{
${ }^{2}$ Enfermeira.Egressa do Mestrado Profissional em Saúde Materno Infantil da UFN E mail:alinegc@yahoo.com.br.

${ }^{3}$ Enfermeira.Egressa do Mestrado Profissional em Saúde Materno Infantil da UFN. Email:lisi_enf@yahoo.com.br.

${ }^{4}$ Enfermeira.Mestranda em Ciências da saúde e da vida da Universidade Franciscana.UFN. Email:stfelvi@yahoo.com.br.

${ }^{5}$ Enfermeiro.Docente do Curso de Enfermagem e do Mestrado Acadêmico em Ciências da Saúde e da Vida da UFN. E-mail:silomar.ilha@ufn.edu.br.

${ }^{6}$ Orientadora.Doutora em Enfermagem. Docente do Curso de Enfermagem e Coordenadora do Mestrado Profissional Saúde Materno Infantil - Universidade Franciscana. E-mail:backesdirce@ufn.edu.br
} 


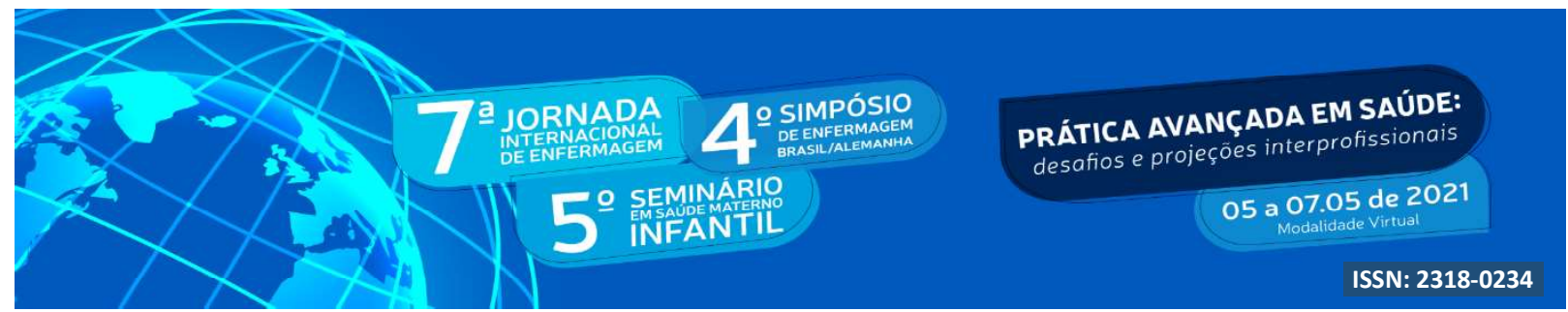

processo legal e privativo do Enfermeiro; e, Sistematização da Assistência de Enfermagem: da concepção à prática assistencial.

\section{Sistematização da Assistência de Enfermagem: processo legal e privativo do Enfermeiro}

Ao serem questionados sobre a sua compreensão de SAE, os Enfermeiros, em sua maioria com mais de dois anos de atuação em uma das unidades intensivas, mencionaram que à mesma se constitui num ato privativo do enfermeiro, o qual ordena a assistência de enfermagem, que embasa legalmente as práticas assistenciais como modelo de trabalho e que se processa em etapas, conforme expresso na fala a seguir: "É uma atividade privativa do enfermeiro [...] ela deve ser realizada por todos os enfermeiros por ser uma resolução, mas nem todas as etapas são cumpridas" (E2).

A SAE, em sua descrição, parece ser adotada, na prática, mais pela sua função legal do que propriamente pelos seu benefícios e vantagens para o processo de cuidado em saúde: "A SAE se constitui em ação privativa do enfermeiro que serve para nortear o trabalho diário da equipe" (E5). "É um conjunto de regras privativas da enfermagem que servem como referencial para o atendimento" (E8). "São ações privativas do enfermeiro as quais norteiam o trabalho diário da equipe" (E9).

A compreensão teórica limitada ficou evidente na medida em que os entrevistados, com frequência, destacaram a SAE pela sua institucionalização legal e como atividade privativa do enfermeiro. Alguns participantes conseguiram transcender a percepção da SAE como dimensão privativa do Enfermeiro e associá-la a equipe de Enfermagem, embora a retratem como método de acompanhamento dos pacientes. Percebe-se, nessa relação, uma percepção reducionista da SAE, tendo em vista que o seu foco está na dimensão biológica do paciente e não no processo de organização, de gestão e de assistência integral e humanizada do processo: "É um método utilizado pelos enfermeiros para auxiliar no processo de acompanhamento de pacientes, como também norteia as atividades de toda equipe de enfermagem” (E7). "É o

${ }^{2}$ Enfermeira.Egressa do Mestrado Profissional em Saúde Materno Infantil da UFN E mail:alinegc@yahoo.com.br.

${ }^{3}$ Enfermeira.Egressa do Mestrado Profissional em Saúde Materno Infantil da UFN. Email:lisi_enf@yahoo.com.br.

${ }^{4}$ Enfermeira.Mestranda em Ciências da saúde e da vida da Universidade Franciscana.UFN. Email:stfelvi@yahoo.com.br.

${ }^{5}$ Enfermeiro.Docente do Curso de Enfermagem e do Mestrado Acadêmico em Ciências da Saúde e da Vida da UFN. E-mail:silomar.ilha@ufn.edu.br.

${ }^{6}$ Orientadora.Doutora em Enfermagem. Docente do Curso de Enfermagem e Coordenadora do Mestrado Profissional Saúde Materno Infantil - Universidade Franciscana. E-mail:backesdirce@ufn.edu.br 


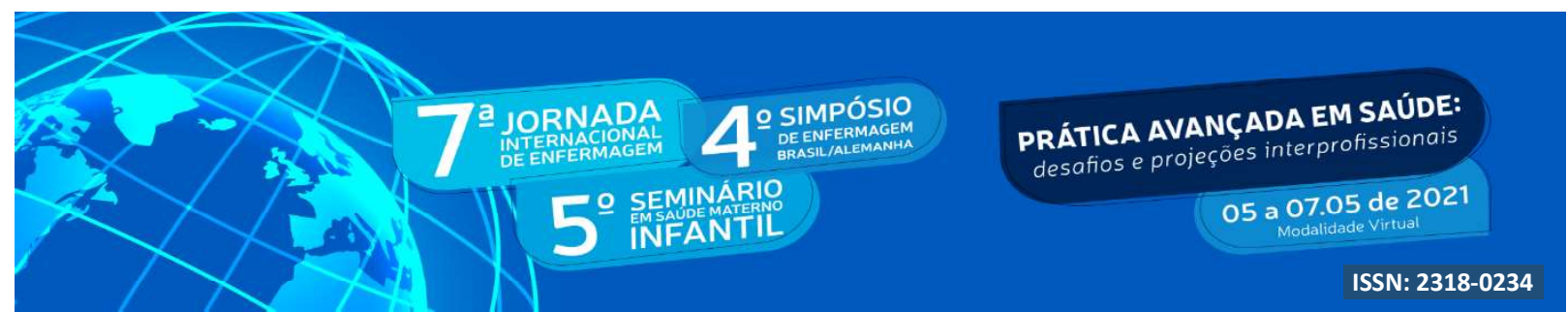

método assistencial que auxilia os enfermeiros no processo de acompanhamento dos pacientes melhorando a qualidade da assistência" (E10). "É o conjunto de regras privativas da enfermagem que servem como referenciais para o atendimento" (E12). "É o instrumento que auxilia os enfermeiros no acompanhamento de pacientes e divide-se em: Histórico de enfermagem, diagnóstico de enfermagem, Plano de assistência, Execução do diagnóstico, Evolução de enfermagem" (E15).

Alguns participantes associaram à SAE ao processo de organização e qualificação da assistência de Enfermagem. Embora demonstrassem que a SAE tem potencial para otimizar, direcionar e potencializar de forma sistemática as ações de cuidado integral, com base em métodos e instrumentos específicos, os entrevistados evidenciam fragilidades relacionadas a apropriação da SAE como método científico, com sistematização sequencial e complementar: "A SAE é uma ferramenta utilizada para direcionar as ações, planejar o cuidado e entender os problemas de forma organizada, embasada legalmente as práticas assistenciais como modelo de trabalho. Suas funções principais são a sistematização e a qualidade da assistência" (E4). "A SAE tem como finalidade organizar e sistematizar as ações do enfermeiro através da metodologia de resolução de problemas" (E14).

Tais fragilidades podem estar associadas a limitada apreensão teórica e/ou aos referenciais reducionistas de intervenção.

\section{CONCUSÃO}

O estudo demonstrou que Enfermeiros das Unidades Intensivas Pediátrica e Neonatal possuem conhecimento teórico superficial sobre a SAE como proposta teórico-metodológica. A compreensão da SAE limita-se, para a maioria, no cumprimento dos preceitos legais e na execução das etapas do diagnóstico e das intervenções de enfermagem.

Embora tenham mencionado que exercem a SAE, na prática assistencial, este processo se limita as etapas do diagnóstico e das intervenções de Enfermagem, as quais se reduzem, na maioria das vezes, em atividades rotineiras e lineares. Embora tenham feito referência às ${ }^{2}$ Enfermeira.Egressa do Mestrado Profissional em Saúde Materno Infantil da UFN E mail:alinegc@yahoo.com.br.

${ }^{3}$ Enfermeira.Egressa do Mestrado Profissional em Saúde Materno Infantil da UFN. Email:lisi enf@yahoo.com.br.

${ }^{4}$ Enfermeira.Mestranda em Ciências da saúde e da vida da Universidade Franciscana.UFN. Email:stfelvi@yahoo.com.br.

${ }^{5}$ Enfermeiro.Docente do Curso de Enfermagem e do Mestrado Acadêmico em Ciências da Saúde e da Vida da UFN. E-mail:silomar.ilha@ufn.edu.br.

${ }^{6}$ Orientadora.Doutora em Enfermagem. Docente do Curso de Enfermagem e Coordenadora do Mestrado Profissional Saúde Materno Infantil - Universidade Franciscana. E-mail:backesdirce@ufn.edu.br 


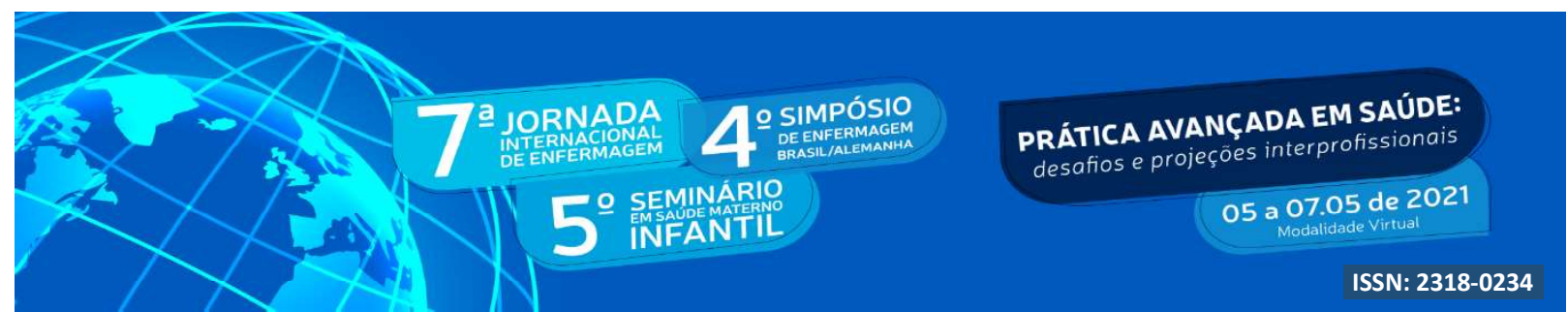

cinco etapas da SAE, em nenhum momento os Enfermeiros se referiram a etapa do Planejamento e da Avaliação do processo de Enfermagem e tão pouco fizeram menção ao referencial teórico adotado no serviço.

Verifica-se, com base no estudo realizado, que a Resolução do COFEN não garante, por si só, a efetivação da SAE na prática assistencial e gerencial dos profissionais de enfermagem. É preciso que gradativamente se discuta abordagens complexas e sistêmicas de intervenção teórico-prática e, sobretudo, estratégias que fomentem a educação continuada e permanente dos profissionais, nos serviços de saúde.

Considera-se como limitações deste estudo a impossibilidade de participação de todos os profissionais Enfermeiros que atuam na unidade intensiva pediátrica. Compreende-se que somente o trabalho em equipe é capaz de ampliar e qualificar a assistência e a gestão de enfermagem. Sugere-se, nessa direção, pesquisas cooperativas entre os diferentes profissionais da equipe de enfermagem, no sentido de ampliar a concepção teórica sobre o tema e garantir a efetivação da SAE em todas as unidades intensivas pediátricas.

Os resultados dessa pesquisa demonstraram que embora sendo exigência legal nos serviços de saúde, a SAE, no senário do estudo, ainda não se efetivou na prática, conforme o desejado. Dessa forma, esta pesquisa pode vir a subsidiar outros estudos na área da enfermagem, a fim de ampliar a sua percepção e fomentar novas estratégias de intervenção que atendam as especificidades, sobretudo, das Unidades Intensivas Pediátricas.

\section{REFERÊNCIAS}

Conselho Federal de Enfermagem (RJ). Resolução COFEN N. 358/2009, de 15 de outubro de 2009: Sistematização da Assistência de Enfermagem e a implementação do Processo de Enfermagem em ambientes, públicos ou privados, em que ocorre o cuidado profissional de Enfermagem. Brasília: COFEN; 2009.

Conselho Federal de Enfermagem (RJ). Resolução COFEN n.272, de 27 de agosto de 2002: Sistematização da Assistência de Enfermagem (SAE) nas instituições de Saúde. Rio de Janeiro: COFEN; 2002.

\footnotetext{
${ }^{2}$ Enfermeira.Egressa do Mestrado Profissional em Saúde Materno Infantil da UFN E mail:alinegc@yahoo.com.br.

${ }^{3}$ Enfermeira.Egressa do Mestrado Profissional em Saúde Materno Infantil da UFN. Email:lisi_enf@yahoo.com.br.

${ }^{4}$ Enfermeira.Mestranda em Ciências da saúde e da vida da Universidade Franciscana.UFN. Email:stfelvi@yahoo.com.br.

${ }^{5}$ Enfermeiro.Docente do Curso de Enfermagem e do Mestrado Acadêmico em Ciências da Saúde e da Vida da UFN. E-mail:silomar.ilha@ufn.edu.br.

${ }^{6}$ Orientadora.Doutora em Enfermagem. Docente do Curso de Enfermagem e Coordenadora do Mestrado Profissional Saúde Materno Infantil - Universidade Franciscana. E-mail:backesdirce@ufn.edu.br
} 


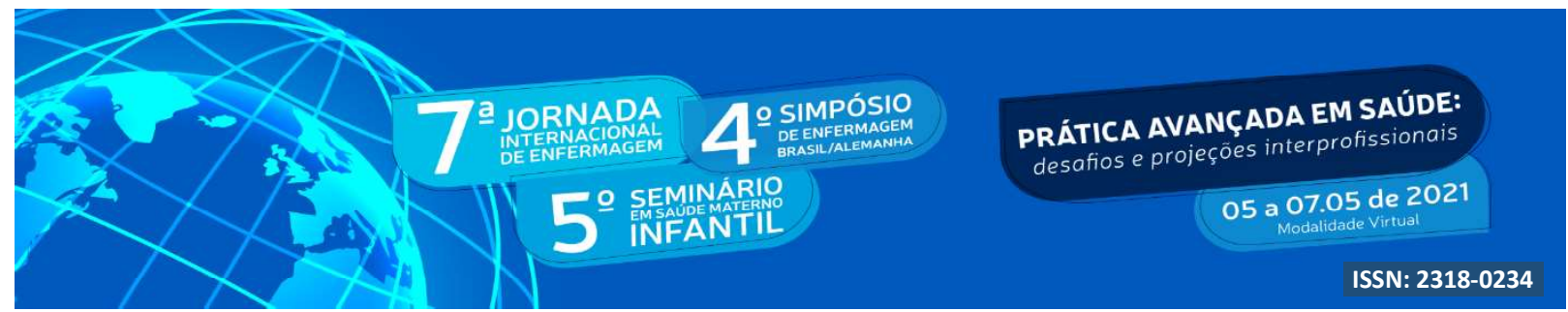

Oliveira KF, Iwamoto HH, Oliveira JF, Almeida DV. Sistematização da Assistência de Enfermagem na Rede Hospitalar de Uberaba MG. Rev Enf Ref [Internet]. 2012 [acessado 08 Jun. 2017]; 3(8):105-14. Available from: http://www.scielo.mec.pt/scielo.php?script=sci_arttext\&pid=S0874-0283201200030001.

Medeiros AL, Santos SR, Cabral RWL. Sistematização da assistência de enfermagem na perspectiva dos enfermeiros: uma abordagem metodológica na teoria fundamentada. Rev Gaúcha Enferm [Internet]. 2012 [acessado 15 dez. 2017]; 33(3):174-81. Disponível em: http://www.scielo.br/scielo.php?script=sci_arttext\&pid=S1983-14472012000300023.

Silva JP, Garanhani ML, Peres MP. Systematization of Nursing Care in undergraduate training: the perspective of Complex Thinking. Rev Latino-Am. Enfermagem [Internet]. 2015 [cited 2017 June 08]; 23(1):59-66. Available from: http://www.scielo.br/pdf/rlae/v23n1/01041169-rlae-23-01-00059.pdf.

Souza NR, Costa BMB, Carneiro DCF, Barbosa HSC, Santos ICRV. Systematization of nursing care: difficulties referred by nurses of a university hospital. Rev enferm UFPE on line [Internet]. 2015 [cited 2017 June 08]; 9(3):7104-10. Available from: https://periodicos.ufpe.br/revistas/revistaenfermagem/article/view/10440/11245.

Tavares TS, Castro AS, Figueiredo ARF, Reis DC. Avaliação da implantação da sistematização da assistência de enfermagem em uma unidade pediátrica. REME Rev Min Enferm [Internet]. 2013 [acessado 08 Jun. 2017]; 17(2): 278-286. Disponível em: http://www.reme.org.br/artigo/detalhes/650.

Brasil. Ministério da Saúde. Conselho Nacional de Saúde. Diretrizes e normas regulamentadoras de pesquisa envolvendo seres humanos. Resolução n. 466, de 12 de dezembro de 2012. Brasília: Ministério da Saúde; 2012.

Minayo MCS. O desafio do conhecimento: pesquisa qualitativa em saúde. 12.ed. Rio Janeiro: Hucitec/Abrasco, 2010.

Gil AC. Como elaborar projeto de pesquisa. 4. ed. São Paulo: Atlas, 2008.

Bardin L. Análise de conteúdo. Lisboa: Edições 70; 2011.

Backes DS. Koerich MS, Nascimento KC, Erdmann AL. Nursing care systematization as a multidimensional and interactive phenomenon. Rev. Latino-Am. Enfermagem [Internet].

${ }^{2}$ Enfermeira.Egressa do Mestrado Profissional em Saúde Materno Infantil da UFN E mail:alinegc@yahoo.com.br.

${ }^{3}$ Enfermeira.Egressa do Mestrado Profissional em Saúde Materno Infantil da UFN. Email:lisi_enf@yahoo.com.br.

${ }^{4}$ Enfermeira.Mestranda em Ciências da saúde e da vida da Universidade Franciscana.UFN. Email:stfelvi@yahoo.com.br.

${ }^{5}$ Enfermeiro.Docente do Curso de Enfermagem e do Mestrado Acadêmico em Ciências da Saúde e da Vida da UFN. E-mail:silomar.ilha@ufn.edu.br.

${ }^{6}$ Orientadora.Doutora em Enfermagem. Docente do Curso de Enfermagem e Coordenadora do Mestrado Profissional Saúde Materno Infantil - Universidade Franciscana. E-mail:backesdirce@ufn.edu.br 


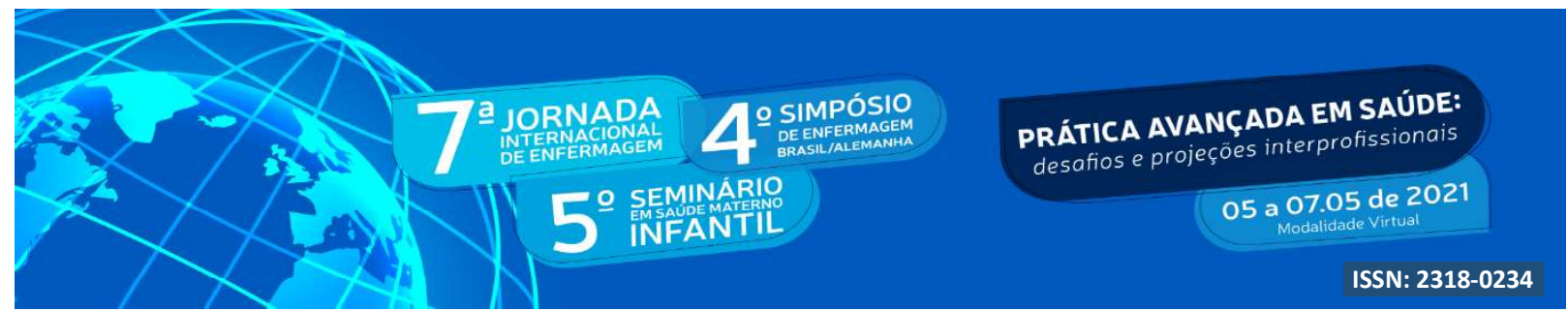

2008 [cited 2017 June 08]; 16(6): 979-85. Available from: http://www.scielo.br/pdf/rlae/v16n6/07.pdf.

Soares MI, Resck ZMR, Terra FS, Camelo SHH. Systematization of nursing care: challenges and features to nurses in the care management. Escola Anna Nery Revista de Enfermagem [Internet]. 2015 [cited 2017 June 08]; 19(1): 47-53. Available from: http://www.scielo.br/pdf/ean/v19n1/en_1414-8145-ean-19-01-0047.pdf.

Silva FSA, Carvalho Filha FSS. Sistematização da assistência de enfermagem a pacientes dialíticos: dificuldades, desafios e perspectivas. Arquivos de Ciências da Saúde [Internet]. 2017 [cited 2018 Jan 08]; 24(2):33-7. Disponível em: http://www.cienciasdasaude.famerp.br/index.php/racs/article/view/599/687.

Boaventura AP, Santos PA, Duran ECM. Conocimiento teórico-práctico del Enfermero del Proceso de Enfermería y Sistematización de Enfermería. Enfermería Global; [Internet]. 2017 [citado 08 Jan 2018]; 16(2):194-205. Disponible en: http://scielo.isciii.es/pdf/eg/v16n46/1695-6141-eg-16-46-00182.pdf.

Grando T, Zuse CL. Dificuldades na instituição da sistematização da assistência de enfermagem no exercício profissional - revisão integrativa. Rev Contexto Saúde [Internet]. 2014 [acesso em 2017 Jun 7]; 4(26):28-35. Disponível em: https://www.revistas.unijui.edu.br/index.php/contextoesaude/article/view/2886/3372.

Silva JP, Aranhani ML, Guariente MHDM. Nursing care systems and complex thought in nursing education: document analysis. Rev. Gaúcha Enferm [Internet]. 2014 [cited 2017 Jun 7]; 35(2):128-34. Available from: http://www.scielo.br/pdf/rgenf/v35n2/1983-1447-rgenf-3502-00128.pdf.

Oliveira CS, Borges MC Social representations of the systematization of the nursing care in the perspective of nurses who take care of children. Rev Gaúcha Enferm [Internet]. 2017 [cited 2018 Jan 08];38(3):e66840.Available from:http://www.seer.ufrgs.br/RevistaGauchade Enfermagem/article/viewFile/66840/44629.

Gutiérrez MGR, Morais SCRV. Systematization of nursing care and the formation of professional identity. Rev Bras Enferm [Internet]. 2017 [cited 2018 Jan 08]; 70(2):455-60. Available from: http://www.scielo.br/pdf/reben/v70n2/0034-7167-reben-70-02-0436.pdf.

\footnotetext{
${ }^{2}$ Enfermeira.Egressa do Mestrado Profissional em Saúde Materno Infantil da UFN E mail:alinegc@yahoo.com.br.

${ }^{3}$ Enfermeira.Egressa do Mestrado Profissional em Saúde Materno Infantil da UFN. Email:lisi_enf@yahoo.com.br.

${ }^{4}$ Enfermeira.Mestranda em Ciências da saúde e da vida da Universidade Franciscana.UFN. Email:stfelvi@yahoo.com.br.

${ }^{5}$ Enfermeiro.Docente do Curso de Enfermagem e do Mestrado Acadêmico em Ciências da Saúde e da Vida da UFN. E-mail:silomar.ilha@ufn.edu.br.

${ }^{6}$ Orientadora.Doutora em Enfermagem. Docente do Curso de Enfermagem e Coordenadora do Mestrado Profissional Saúde Materno Infantil - Universidade Franciscana. E-mail:backesdirce@ufn.edu.br
} 